\title{
Symbolic Solution of Kepler's Generalized Equation
}

\author{
Juan Félix San-Juan ${ }^{1}$ and Alberto Abad ${ }^{2}$ \\ 1 Universidad de La Rioja, 26004 Logroño, Spain \\ juanfelix.sanjuan@dmc.unirioja.es, \\ 2 Grupo de Mecánica Espacial, \\ Universidad de Zaragoza, 50009 Zaragoza - Spain abad@posta.unizar.es
}

\begin{abstract}
Kepler's generalized equation is a transcendental nonlinear equation that must be solved in order to compute the position and velocity of an artificial satellite at any instant $t$. In this paper, we propose a method to solve analytically that equation. The method is based on the properties of non canonical Lie transformations and, under certain conditions, it is shown that it gives better results than numerical methods.
\end{abstract}

\section{Introduction}

The design of spatial missions requires the development of analytical theories in order to place an artificial satellite in orbit around a planet. These theories are particularly appreciated by mission analysis engineers at space agencies.

ATESAT 121213 is a symbolic software that automatically generates analytical theories for the orbital motion of satellites. This software makes extensively use of Lie transformations [6] applied to the original problem (a mass point under the gravitational field of a planet) in order to generate automatically the symbolic expressions of the equations of motion as well as the C-code to compute the position and velocity (ephemeris) for the satellite. It is currently implemented at the Centre National d'Etudes Spatials (CNES) of Toulouse (France) and is used in mission analysis for orbiters around the Earth and Mars. Among these transformations used in ATESAT, let us mention the elimination of the parallax [8], the elimination of the perigee [5], and the Krylov-Bogoliuvob-Mitropolsky (KBM) method 910. The description of these transformations is beyond the scope of this paper and will not be included here; more details can be found in the original papers or in 23 and references therein. Let us mention, that after the application of this set of transformations, the problem is greatly simplified and reduced to a perturbed harmonic oscillator [3]. In this way, it is possible to reach high orders of precision, with the advantage that, once the theory is obtained, the evaluation of the position or velocity vectors is just a matter of evaluating a time function (see e.g. [4]).

When we try to solve by analytical means, this new problem, we meet a transcendental equation whose solution must be obtained. This equation is similar to the Kepler's classical equation in the two-body problem, and this is why 
the new equation is called Kepler's generalized equation. These equations are usually solved numerically, but for some specific values of the parameters, the convergence is poor and some subtle details must be taken into account.

Let us remind that the Kepler's classical equation is

$$
\ell=n(t-T)=E-e \sin E,
$$

where it relates the mean anomaly $\ell$, or the time $t$, to the eccentric anomaly $E$ and the eccentricity $e$ of the orbit. The solution of this equation, that is, finding $E$ for a given value of time (or $\ell$ ) is an operation that we have to perform each time we want to know the position (or the distance) of the satellite at any instant $t$. This equation cannot be directly inverted in terms of simple functions because it is transcendental in $E$. The only way to express analytically $E$ in terms of $\ell$ is by means of a power series expansion of the eccentricity that diverges for values of $e>0.6627434$. Many algorithms have been developed to solve Kepler's equation due to its relevance for Celestial Mechanics and Astrodynamics.

A way to solve it is to apply Lagrange's inversion theorem: Let $y$ be defined as a function of $x$ in terms of a parameter $\alpha$ by $y=x+\alpha \phi(y)$, Lagrange's inversion theorem ensures that $y$ can be expressed as a function of $x$ as a power series in $\alpha$. In [7] we find a more general formulation of the Lagrange's inversion theorem, based on non-canonical Lie transformations, very useful to solve Kepler's equation.

For our part, in this Note, we present an analytical method to solve Kepler's generalized equation. This equation coincides with the classical one when the small parameter $\epsilon$ of the perturbation expansion in the orbit is $\epsilon=0$. Our approach is a method to invert symbolically Kepler's generalized equation. The solution that we propose is completely formulated in terms of non-canonical Lie transformations. We apply our algorithm to some practical examples, showing that the results obtained with our method are better than those obtained with numerical ones.

\section{A First Order Solution of the Main Problem of the Artificial Satellite}

The Keplerian orbit of an artificial satellite is perturbed by the non sphericity of the planet. It is modeled by the value of its potential harmonics. The most important effect an the orbit is generally due to the oblateness of the planet, and the corresponding harmonic is the second order zonal harmonic $J_{2}$. The model of the orbit of a satellite perturbed only by $J_{2}$ is called main problem of the satellite.

The most adequate set of variables to formulate this problem are the Whittaker's or polar-nodal variables $(r, \theta, \nu, R, \Theta, N)$, where $r$ is the radial distance from the planet's mass center to the satellite, $\theta$ is the argument of latitude, and $\nu$ is the argument of the ascending node. The variables $R, \Theta, N$ are the conjugate momenta to the coordinates $r, \theta, \nu$, respectively (see Figure 1). 


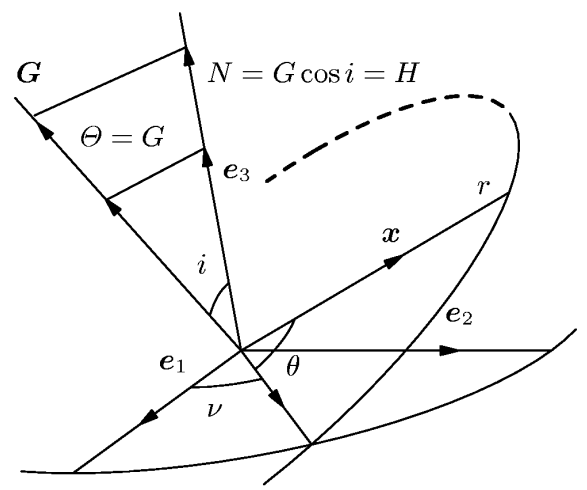

Fig. 1. Polar-nodal coordinates

The Hamiltonian of the main problem expressed in Whittaker's or polarnodal variables (see Deprit, 8], for details) is

$$
\mathcal{H}\left(r, \theta,{ }_{-}, R, \Theta, N\right)=\mathcal{H}_{0}+\epsilon \mathcal{H}_{1},
$$

where

$$
\mathcal{H}_{0}=\frac{1}{2}\left(R^{2}+\frac{\Theta^{2}}{r^{2}}\right)-\frac{\mu}{r}, \quad \mathcal{H}_{1}=\frac{\mu}{r}\left(\frac{\alpha}{r}\right)^{2} P_{2}(\sin i \sin \theta),
$$

$\mu$ is the gravitational constant, $\alpha$ is the equatorial radius of the planet, $i$ is the angle of intersection between the orbital plane and the equator and the parameter $\epsilon$ represents the oblateness $J_{2}$ of the Earth.

In [3] we show a complete second order integration of this problem. However, the expression involved in such theory is very extremely large and we only present here the first order. First, we apply two canonical transformations that reduce to one the number of degrees of freedom. After that, the Krylov-BogoliuvovMitropolski technique is applied to integrate the problem.

The elimination of the parallax [8] is the first canonical transformation implemented. It reduces the complexity of the subsequent calculations when going up to higher orders. The second canonical transformation is the elimination of the perigee [5]. It removes the argument of the perigee from the Hamiltonian giving the expression

$$
\mathcal{H}\left(r,,_{-}, R, \Theta, N\right)=\frac{1}{2}\left(R^{2}+\frac{\Theta^{2}}{r^{2}}\right)-\frac{\mu}{r}+\epsilon \frac{\alpha^{2} \mu^{2}}{\Theta^{2}}\left(\frac{1}{2}-\frac{3}{4} \sin ^{2} i\right) \frac{1}{r^{2}},
$$

where the argument of the latitude $\theta$ does not appear and the number of degrees of freedom is reduced to one.

Changing the variables $r, d r / d t$ and the time $t$ for two new variables $u, v$ and a new independent variable $s$ respectively, defined by

$$
u=\frac{1}{r}-\beta, \quad r^{2} \frac{d s}{d t}=\Theta, \quad v=\frac{d u}{d s},
$$

we convert Hamilton's equation into the perturbed harmonic oscillator 


$$
\frac{d^{2} u}{d s^{2}}+u=\epsilon\left(\mathcal{K}_{1}+\mathcal{K}_{2} u\right)
$$

where

$$
\mathcal{K}_{1}=-\frac{2 \alpha^{2} \mu^{3}}{\Theta^{6}}\left(\frac{1}{2}-\frac{3}{4} \sin ^{2} i\right), \quad \mathcal{K}_{2}=-\frac{2 \alpha^{2} \mu^{2}}{\Theta^{4}}\left(\frac{1}{2}-\frac{3}{4} \sin ^{2} i\right)
$$

are constants.

The Krylov-Bogoliubov-Mitropolsky method 9], 10] assumes an asymptotic expansion of the solution of a perturbed harmonic oscillator in the form

$$
u=\delta \cos f+\sum_{i \geq 1} \frac{\epsilon^{i}}{i !} u_{i}(\delta, f)
$$

where $u_{i}$ are $2 \pi$-periodic functions in $f$, and the relation of $\delta$ and $f$ with time $s$ is given by

$$
\frac{d \delta}{d s}=\sum_{i \geq 0} \frac{\epsilon^{i}}{i !} A_{n}(\delta), \quad \frac{d f}{d s}=\sum_{i \geq 1} \frac{\epsilon^{i}}{i !} B_{n}(\delta)
$$

In our case

$$
\frac{d \delta}{d s}=0, \quad \frac{d f}{d s}=n_{f}=1-\epsilon \frac{\mathcal{K}_{2}}{2} .
$$

Combining the relations of $f$ with $s$ and $s$ with $t$ we obtain the differential relation between $f$ and $t$. To integrate this relation, an auxiliary variable $E$, which is called the eccentric anomaly, is defined via the equations

$$
\cos f=\frac{\sqrt{1-e^{2}} \sin E}{1-e \cos E}, \quad \sin f=\frac{\cos E-e}{1-e \cos E} .
$$

Finally, we obtain

$$
\ell=E-e \sin E+\epsilon \frac{\mathcal{K}}{\left(1-e^{2}\right)^{3}}\left[\left(1+\frac{e^{2}}{2}\right) E-2 e \sin E+\frac{e^{2}}{4} \sin 2 E\right]
$$

where $\ell=n(t-T)$, with a constant $n=n_{f} \sqrt{\mu / a^{3}}$, slightly different from the mean motion and a constant $\mathcal{K}=\frac{\alpha^{2}}{\mu^{2} a^{2}}\left(2-3 \sin ^{2} i\right)$. Equation (4) is Kepler's generalized equation.

A more detailed version of this process can be found in [3]. We show there all the expressions of the main problem up to the order two. The same process can be applied to greater orders and more complex models considering not only the oblateness of the planet, but any zonal potential harmonic of the planet. In all the cases, we obtain a generalized Kepler's equation of the form

$$
\ell=E-e \sin E+\sum_{i>0} \frac{\epsilon^{i}}{i !} G_{i}(e, E)
$$




\section{Inversion of Series Using Non-canonical Lie Transformations}

Definition 1. We call non-canonical Lie transformation of generator $\boldsymbol{W}(\boldsymbol{x} ; \epsilon)$ the solution

$$
\varphi:(\boldsymbol{y}, \epsilon) \rightarrow \boldsymbol{x}(\boldsymbol{y}, \epsilon)=\sum_{n \geq 0} \frac{\epsilon^{n}}{n !} \boldsymbol{x}_{n}(\boldsymbol{y}): \mathbb{R}^{m} \times \mathbb{R} \rightarrow \mathbb{R}^{m},
$$

of the ordinary differential equation

$$
\frac{d \boldsymbol{x}}{d \epsilon}=\boldsymbol{W}(\boldsymbol{x} ; \epsilon)=\sum_{n \geq 0} \frac{\epsilon^{n}}{n !} \boldsymbol{W}_{n+1}(\boldsymbol{x}), \quad \boldsymbol{x}(\boldsymbol{y}, 0)=\boldsymbol{y} .
$$

The following propositions describe the two main properties of a Lie transformation.

Proposition 1. The expression

$$
\varphi^{*} \boldsymbol{F}(\boldsymbol{y} ; \epsilon)=\boldsymbol{F}(\boldsymbol{x}(\boldsymbol{y}, \epsilon) ; \epsilon)=\sum_{n \geq 0} \frac{\epsilon^{n}}{n !} \boldsymbol{F}_{0, n}(\boldsymbol{y}),
$$

resulting from the application of the Lie transformation $\varphi$ to the analytical function

$$
\boldsymbol{F}(\boldsymbol{x} ; \epsilon)=\sum_{n \geq 0} \frac{\epsilon^{n}}{n !} \boldsymbol{F}_{n, 0}(\boldsymbol{x})
$$

is obtained by using the iterative algorithm called Lie triangle, given by the expressions

$$
\boldsymbol{F}_{n, p}=\boldsymbol{F}_{n+1, p-1}+\sum_{i+j=n} \frac{n !}{i ! j !}\left(\nabla_{\boldsymbol{x}} \boldsymbol{F}_{i, p-1} \cdot \boldsymbol{W}_{j+1}\right)
$$

Proposition 2. The inverse of a non-canonical Lie transformation $\varphi$ whose explicit expression is given by (6) is a non-canonical Lie transformation whose generator $\boldsymbol{V}(\boldsymbol{y} ; \epsilon)$ is given, order by order, by the expressions

$$
\begin{aligned}
& \boldsymbol{V}_{1}=-\boldsymbol{x}_{1}, \\
& \boldsymbol{V}_{n+1}=-\boldsymbol{x}_{n+1}-\sum_{i=0}^{n-1}\left(\begin{array}{c}
n \\
i
\end{array}\right)\left(\nabla \boldsymbol{y} \boldsymbol{x}_{j} \cdot \boldsymbol{V}_{i+1}\right), \quad n \geq 1 .
\end{aligned}
$$

As we can see in the previous definition and properties, a Lie transformation is a near identity transformation that can be characterized indistinctly by its explicit expression or its generator. The Lie triangle applied to the function $\boldsymbol{F}(\boldsymbol{x}, \epsilon)=\boldsymbol{x}$ obtains the explicit expression from the generator.

A detailed description of the properties and applications of these transformations can be found in Kamel [12], Henrard [11] and Deprit [6]. 


\section{Inversion of Kepler's Generalized Equation}

To illustrate the inversion of Kepler's generalized equation we use (4) as a starting point. Like in the Kepler's classical equation, we look for an expression of the eccentric anomaly $E$ in terms of the mean anomaly $\ell$ as an expansion in powers of the eccentricity $e$. To this aim, we expand (41) in powers of $e$ up to the sixth order in the form

$$
\begin{aligned}
\ell= & (1+\mathcal{K} \epsilon) E+e(-\sin E-\epsilon 2 \mathcal{K} \sin E)+\frac{e^{2}}{2} \epsilon \mathcal{K}\left(7 E+\frac{1}{2} \sin 2 E\right) \\
& +\frac{e^{3}}{6} \epsilon(-36 \mathcal{K} \sin E)+\frac{e^{4}}{24} \epsilon \mathcal{K}(180 E+18 \sin 2 E)+\frac{e^{5}}{120} \epsilon(-1440 \mathcal{K} \sin E) \\
& +\frac{e^{6}}{720} \epsilon \mathcal{K}(9360 E+1080 \sin 2 E)+\mathcal{O}\left(e^{7}\right),
\end{aligned}
$$

where the coefficients of the expansion are expansions in powers of the small parameter $\epsilon$ up to the the order of the theory, in this case, order one. We choose order six in the expansion in powers of $e$ to obtain the machine precision in the numerical evaluation of this approximation.

Using the arithmetic of power series, we divide it by $(1+\mathcal{K} \epsilon)$ and obtain the expression

$$
\begin{aligned}
\ell^{\prime}= & E+e(-\sin E-\epsilon \mathcal{K} \sin E)+\frac{e^{2}}{2} \epsilon \mathcal{K}\left(7 E+\frac{1}{2} \sin 2 E\right) \\
& +\frac{e^{3}}{6} \epsilon(-36 \mathcal{K} \sin E)+\frac{e^{4}}{24} \epsilon \mathcal{K}(180 E+18 \sin 2 E)+\frac{e^{5}}{120} \epsilon(-1440 \mathcal{K} \sin E) \\
& +\frac{e^{6}}{720} \epsilon \mathcal{K}(9360 E+1080 \sin 2 E)+\mathcal{O}\left(e^{7}\right),
\end{aligned}
$$

where $\ell^{\prime}=\ell /(1+\mathcal{K} \epsilon)$.

Equation (12) has the form of an explicit non canonical Lie transformation. An application of the second proposition gives the generator of the inverse transformation. Finally, the first proposition gives the explicit expression of the inverse of (12), that is equal to

$$
\begin{aligned}
E= & \ell^{\prime}+e\left(\sin \ell^{\prime}+\epsilon \mathcal{K} \sin \ell^{\prime}\right)+\frac{e^{2}}{2}\left[\sin 2 \ell^{\prime}+\epsilon \mathcal{K}\left(-7 \ell^{\prime}+\frac{3}{2} \sin 2 \ell^{\prime}\right)\right] \\
& +\frac{e^{3}}{6}\left[\left(\frac{3}{4} \sin \ell^{\prime}+\frac{9}{4} \sin 3 \ell^{\prime}\right)+\epsilon \mathcal{K}\left(-21 \ell^{\prime} \cos \ell^{\prime}+\frac{27}{2} \sin \ell^{\prime}+\frac{9}{2} \sin 3 \ell^{\prime}\right)\right] \\
& +\frac{e^{4}}{24}\left[\left(-4 \sin 2 \ell^{\prime}+8 \sin 4 \ell^{\prime}\right)+\epsilon \mathcal{K}\left(-180 \ell^{\prime}-84 \ell^{\prime} \cos 2 \ell^{\prime}+32 \sin 2 \ell^{\prime}\right.\right. \\
& \left.\left.+20 \sin 4 \ell^{\prime}\right)\right]+\frac{e^{5}}{120}\left[\left(\frac{5}{8} \sin \ell^{\prime}-\frac{405}{16} \sin 3 \ell^{\prime}+\frac{605}{16} \sin 5 \ell^{\prime}\right)\right. \\
& +\epsilon \mathcal{K}^{\prime}\left(-\frac{1695}{2} \ell^{\prime} \cos \ell^{\prime}-\frac{945}{2} \ell^{\prime} \cos 3 \ell^{\prime}+\frac{3759}{8} \sin \ell^{\prime}+\frac{2025}{16} \sin 3 \ell\right.
\end{aligned}
$$




$$
\begin{aligned}
& \left.\left.+\frac{1875}{16} \sin 5 \ell^{\prime}\right)\right]+\frac{e^{6}}{720}\left[\left(16 \sin \ell^{\prime}-192 \sin 4 \ell^{\prime}+243 \sin 6 \ell^{\prime}\right)\right. \\
& +\epsilon\left(-9360 \ell^{\prime}-4560 \ell \cos 2 \ell^{\prime}-3360 \ell^{\prime} \cos 4 \ell^{\prime}+\frac{3105}{2} \sin 2 \ell^{\prime}\right. \\
& \left.\left.+648 \sin 4 \ell^{\prime}+\frac{1701}{2} \sin 6 \ell^{\prime}\right)\right] .
\end{aligned}
$$

Eventually, we will go from $\ell^{\prime}$ to $\ell$ using again the arithmetic of power series and the relation $\ell^{\prime}=\ell /(1+\mathcal{K} \epsilon)$, then we will obtain the mean anomaly in terms of the true anomaly in Kepler's generalized equation, that in the general case will have the expression

$$
E=\ell+\sum_{i \geq 1} \frac{e^{i}}{i !} E_{i}(\ell ; \epsilon)=\sum_{i \geq 1} \frac{e^{i}}{i !} \sum_{n \geq 0}\left(\frac{\epsilon^{n}}{n !} \phi_{n}(\ell)\right)
$$

In our particular case, we have

$$
\begin{aligned}
E_{1}= & \ell-\epsilon \mathcal{K} \ell \\
E_{2}= & \sin \ell+\epsilon \mathcal{K}(-\ell \cos \ell+\sin \ell) \\
E_{3}= & \sin 2 \ell+\epsilon \mathcal{K}\left(-7 \ell-2 \ell \cos 2 \ell+\frac{3}{2} \sin 2 \ell\right), \\
E_{4}= & -\frac{3}{4} \sin \ell+\frac{9}{4} \sin 3 \ell \\
& +\epsilon \mathcal{K}\left(-\frac{81}{4} \ell \cos \ell-\frac{27}{4} \ell \cos 3 \ell+\frac{27}{2} \sin \ell+\frac{9}{2} \sin 3 \ell\right), \\
E_{5}= & -4 \sin 2 \ell+8 \sin 4 \ell \\
& +\epsilon \mathcal{K}(-180 \ell-72 \ell \cos 2 \ell-32 \ell \cos 4 \ell+32 \sin 2 \ell+20 \sin 4 \ell), \\
& 5 \\
E_{6}= & \sin \ell-\frac{405}{16} \sin 3 \ell+\frac{625}{16} \sin 5 \ell \\
& +\epsilon \mathcal{K}\left(\frac{6785}{8} \ell \cos \ell-\frac{6345}{16} \ell \cos 3 \ell+\frac{3125}{16} \ell \cos 5 \ell\right. \\
& \left.+\frac{3795}{8} \sin \ell+\frac{2025}{16} \sin 3 \ell+\frac{1875}{16} \sin 5 \ell\right), \\
& \\
&
\end{aligned}
$$

\section{Numerical Evaluation}

In order to compute the ephemeris of a satellite using the model previously described we have to solve numerically the Kepler's generalized equation. The 
classical solution for Kepler's equation is to apply Newton's method by the iterative formula

$$
E_{i+1}=E_{i}-\frac{f\left(E_{i}\right)}{f^{\prime}\left(E_{i}\right)},
$$

where $f(E)=E-\ell-e \sin E$, but methods to improve the convergence of this equation appear frequently in the literature due to the relevance of the equation in the field of orbital motion.

We may apply the same method to Kepler's generalized equation, with a function $f(E)$ defined by

$$
f(E)=E-\ell-e \sin E+G(e, E ; \epsilon) .
$$

When we apply this method there are convergence problems that lead to erroneous determinations of the position of the satellite.

We may use the formula (13) to evaluate Kepler's generalized equation when eccentricity values are small. For instance, in the real case of a Spot satellite $(e=0.01)$, in the computation of ephemeris for a period of 60 days, the error generated by the use of the analytical approximation instead of the numerical method was about $6 \times 10^{-5}, 6 \times 10^{-7}, 6 \times 10^{-9}, 8 \times 10^{-11}, 1 \times 10^{-12}, 1 \times 10^{-13}$ for a development in $e$ of order $1,2,3,4,5,6$, respectively.

Acknowledgment. This work has been supported in part by the Ministerio de Educación y Ciencia (PB98-1576, BFM2002-03157) and the Department of Space Mathematics of Centre National d'Etudes Spatiales (France).

\section{References}

1. Abad, A. and San Juan, J. F.: ATESAT: software tool for obtaining automatically ephemeris from analytical simplifications. Cahiers du Centre Européen de Géodynamique et de Séismologie 10 (1995) 93-98

2. Abad, A., Elipe, A., Palacián, J. and San-Juan, J. F.: ATESAT: A Symbolic Processor for Artificial Satellite Theory. Mathematics and Computers in Simulation 45, (1998) 497-510.

3. Abad, A., J.F. San Juan and A. Gavín.: Short term evolution of artificial satellites. Celestial Mechanics \& Dynamical Astronomy 79(4) (2001) 277-296

4. Abad, A., Elipe, A., San-Juan, J. F. and Serrano, S.: Is Symbolic Integration better than Numerical Integration in Satellite Dynamics?. Accepted Applied Mathematics Letters, (2003).

5. Alfriend, K. T. and Coffey, S. L.: Elimination of the Perigee in Satellite Problem. Celestial Mechanics 32 (1984) 163-172

6. Deprit, A.: Canonical Transformations Depending on a Small Parameter. Celestial Mechanics 1 (1969) 12-30

7. Deprit, A.: Note on Lagrange's inversion formula. Celestial Mechanics 20 (1979) 325-327

8. Deprit, A.: The elimination of the parallax in Satellite Theory. Celestial Mechanics 24 (1981) 111-153 
9. Krylov, N. and Bogoliubov, N. N.: Introduction to Nonlinear Mechanics. Princeton University Press, Princeton N.Y. (1947) KB47

10. Bogoliubov, N. N. and Mitropolsky, Y. A.: Asymptotic Method in the Theory of Nonlinear Oscillations. Gordon and Breach, New York (1961)

11. Henrard, J.: On a perturbation theory using Lie Transform. Celestial Mechanics 3 (1970) 107-120

12. Kamel, A. A.: Perturbation methods in the theory of nonlinear oscillations. Celestial Mechanics 3 (1970) 90-106

13. San Juan, J. F.: Manipulación algebraica de series de Poisson. Aplicación a la teoría del satélite artificial. Ph.D. thesis (1996), University of Zaragoza 